\title{
Pseudoknots in a Homopolymer
}

\author{
A. Kabakçıŏlu and A. L. Stella ${ }^{\dagger}$ \\ INFM - Dipartimento di Fisica, Università di Padova, I-35131 Padova, Italy \\ † Sezione INFN, Università di Padova, I-35131 Padova, Italy
}

(Dated: November 2, 2018)

\begin{abstract}
After a discussion of the definition and number of pseudoknots, we reconsider the self-attracting homopolymer paying particular attention to the scaling of the pseudoknot number $\left(N_{p k}\right)$ at different temperature regimes in two and three dimensions. We find that, although the total number of pseudoknots is extensive at all temperatures, the number of those forming between the two halves of the chain diverges logarithmically at (both dimensions) and below ( $2 \mathrm{~d}$ only) the $\theta$-temparature. We later introduce a simple model that emphasizes the role of pseudoknot formation during collapse. The resulting phase diagram involves swollen, branched and collapsed homopolymer phases with transitions between each pair.

PACS numbers: 36.20.Ey, 87.15.Aa, 05.40.-a
\end{abstract}

\section{INTRODUCTION}

Fueled mainly by the advance in RNA structure determination techniques, recently there has been growing interest in understanding and predicting formation of pseudoknots in RNAs. A pseudoknot $(\mathrm{PK})$ is not a true knot in the conventional sense. It is a simpler construct generated by a polymer's self-contacts (see the definition below), therefore is encountered more frequently. Unlike true knots which are problematic for the DNA and occur occasionally in shorter biomolecules, PKs are the tertiary structure of the folded RNAs. They are known to exist in almost all RNA classes including transfer, messanger, ribosomal, viral, catalytic and self-splicing RNAs (see reviews [1]). A recent analysis found that they account for up to $30 \%$ of the bound base pairs in $\mathrm{G}+\mathrm{C}$ rich $\mathrm{RNA}$ sequences 2]. In addition to stabilizing the fold, PKs are believed to assume functional roles, such as mediating the binding of the proteins they encode [3] , labeling functionally important positions on the coding regions of the mRNA sequence [4, 5], mediating frameshifting [6], etc.

Being a more elementary topological formation than knots, PKs are relatively amenable to numerical investigation. Nevertheless, most of the earlier computational tools and recent theoretical work on RNA structure prediction take into consideration only those configurations without PKs [7, 8, 9]. This is mostly because ignoring PKs results in a 'nested' set of equations and, as a consequence, allows efficient dynamic programming techniques. The drawback is that their success is limited to secondary structure prediction only. And even then, with limited accuracy due, partially, to a necessary reorganization of the secondary structure contacts to accomodate the PKs. More recently there appeared computational 10, 11] and theoretical 12, 13, 14, 15] studies that include PKs into RNA structure prediction algorithms. Pilsbury et.al. suggest a diagrammatic expansion to perturbatively take into account the PKs 12]. A recent study by Baiesi et.al. [13] takes a more physical look on RNA denaturation. They model the RNA as a ho- mopolymer traversing a two-tolerant walk on the FCC lattice and consider walks both with and without PKs [16]. They conclude that the sharp second-order denaturation transition observed when PKs are allowed gives way to a smooth crossover upon their exclusion. This result emphasizes the thermodynamic relevance of PKs. Lucas et.al. 14 consider lattice homopolymers again to argue that the denaturation transition between the pseudoknotted state and the open state is continuous. Another two-tolerant trail model with pseudoknots and with a native state consisting of a single hairpin is argued to denaturate through a first-order transition [15]. Studying the interplay between the PKs and the transition thermodynamics within the homopolymer context is a prerequisite for a deeper understanding of the physics of RNA pseudoknots.

Folding experiments on RNAs suggest that it is physically more appropriate to attribute a different binding energy to the contacts that form a PK 17]. This energy can be tuned by changing the $\mathrm{Mg}^{+2}$ concentration in the solvent. Unless this energy is prohibitively high (as in the case of very low $\mathrm{Mg}^{+2}$ concentration), calculating the Boltzmann weights necessitates identifying PKs for an arbitrary configuration. As we shall see below, this task, though may be easier in native RNA configurations, is nontrivial for an arbitrary polymer.

Accordingly, our goal throughout this paper will be to explore the thermodynamic role assumed by the PKs in the well-known context of homopolymer collapse by:

1. providing an analytic definition for the PK number (Section II),

2. investigating the scaling properties of the PK number in various regimes of the homopolymer collapse (Section III),

3. generalizing the Hamiltonian for the self-attracting homopolymer to include an arbitrary penalty for PKs and obtaining the corresponding phase diagram (Section IV).

We hope that our results will provide a better understanding to the nature of the PKs and a new perspective to the homopolymer collapse transition by locating it in a 
more general framework that also includes the branched polymers and their collapse transition.

\section{COUNTING PSEUDOKNOTS}

An arbitrary configuration of a polymer chain can be encoded as a contact map [18] which is a binary symmetric matrix. The contact map, in turn, can be represented graphically by an 'arc diagram' as follows: Imagine stretching the polymer into a horizontal straight line by pulling from the two ends. Next, connect each pair of monomers that were originally in contact by a semicircular arc on the upper half plane (the diagram is drawn on a plane even though the polymer may be embedded in arbitrary dimensions).

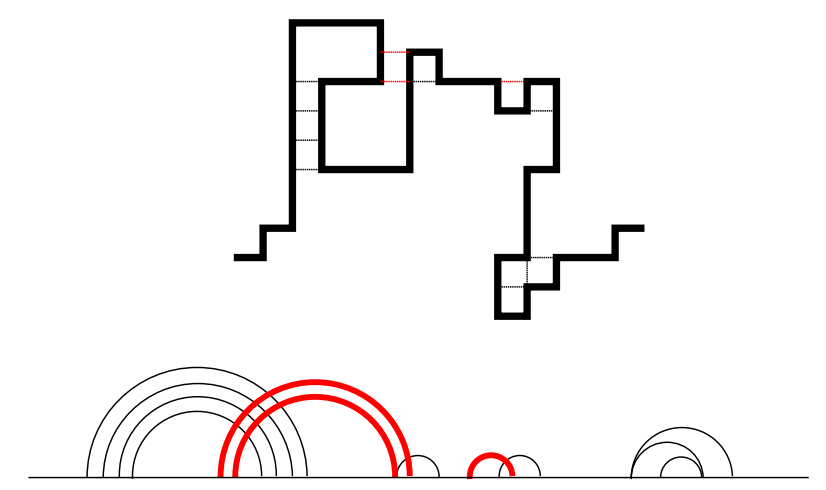

FIG. 1: A sample SAW on a square lattice and its selfcontacts (edges not traversed by the walk that connect two visited nearest-neighbor lattice sites) together with the corresponding arc diagram and its non-unique minimal contact set (shown in bold) to be removed in order to reduce it to a planar diagram.

The diagram is said to be planar if no two arcs cross each other. This is equivalent to having no PKs. In the opposite case, a selective treatment of the PKs primarily requires identification of their number. Since the definition involves crossings in the arc diagram, one might be inclined to simply count the number of crossings. However, this does not make physical sense, because an arbitrary number of crossings may be generated by the addition of a single contact, as is obvious from Fig [1 Instead we need a quantity that reflects the number of contacts that are responsible for the pseudoknots. Accordingly, we define $N_{p k}$, the PK number, as the minimum number of arcs that need to be removed to reach a planar diagram. The same definition was recently adopted in another study on RNA pseudoknot prediction [2]. We stress that the choice of this minimal set is in general not unique. In Fig 1 we show a SAW in two dimensions and the corresponding arc diagram, where one possible choice of a minimal set of arcs which, when removed, leave a planar diagram is drawn in bold. Therefore, although one can talk about a unique PK number, labeling some of the contacts as PK forming contacts requires adopting an extra arbitrary convention. In this study, we avoid this by resting our results on the mere knowledge of the number of PKs.

Our first observation is that calculating $N_{p k}$ exactly for an arbitrary arc diagram belongs to a class of problems known as NP-complete, implying that there's no known deterministic polynomial-time algorithm for calculating $N_{p k}$ 19. We prove this by mapping our problem to one of the six well-known problems in computer science that are shown to be NP-complete, namely the 'vertexcovering' problem. For a recent review of the vertexcovering problem in the statistical physics context see 20]. The mapping is easily established by representing each arc by a vertex and drawing edges between pairs of vertices corresponding to crossing arcs (see Fig 2). The

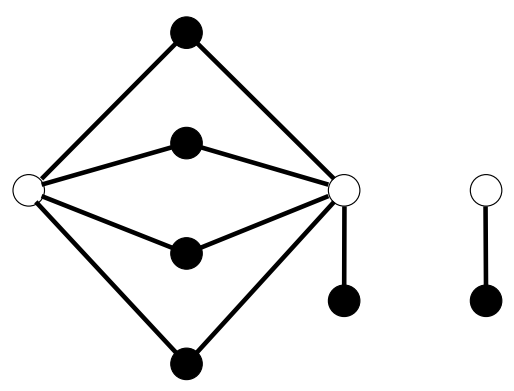

FIG. 2: The resulting graph for the arc diagram in Fig 1 after the mapping to the vertex-covering problem. Empty vertices correspond to the solution represented by bold arcs in Fig 1

vertex-covering problem on the obtained graph (known as the incompatibility graph) amounts to finding a minimal set of vertices which, when labelled, results in labelling at least one end of every edge. In other words, erasing those vertices alone together with the edges sprouting from each is sufficient to get rid of all the edges in the graph. Since every edge reflects a crossing and every vertex an arc in the original arc diagram, eliminating those arcs corresponding to the minimal vertex set obviously results in a planar diagram, i.e., the size of this minimal set is equal to $N_{p k}$. Since we need to calculate $N_{p k}$ for many polymer configurations in our study, it is important to be aware that an exact treatment requires $\mathrm{CPU}$ times exponential in the number of arc-crossings. The above mapping is a different and simpler statement of NP-completeness than an earlier proof that a large class of RNA secondary structure prediction algorithms based on free energy minimization with pseudoknots are NPcomplete 21]. Also note that when restricted to a certain subset of polymer configurations (typically generated in an iterative manner), the problem can be solved in polynomial time. Efficient algorithms on such restricted configurations have been recently utilized to predict RNA pseudoknots 2, 10, 12].

What is the expected value of $N_{p k}$ for a polymer? We would first like to provide some insight for the reader by 
calculating $N_{p k}$ for a random arc diagram: Consider $N_{c}$ randomly chosen distinct pairs of points on a line segment and connect them by arcs as in Fig \. The probability that $m$ randomly placed arcs do not intersect (i.e., form a planar graph) is

$$
P_{\text {planar }}(m) \sim 1 /(m !) .
$$

Proof by induction: the $m^{\text {th }}$ non-intersecting arc to be placed will see the polymer divided into $m$ compartments, such that, if the arc is placed with its legs resting on different compartments, it has to cross at least one other arc. Then, the probability that the $m^{\text {th }}$ arc doesn't cross (given the first $m-1 \operatorname{arcs}$ ) is roughly $1 / m$, i.e., the probability that the two points fall into the same bin. Eq.(1) follows by induction.

Then, such a subset of $m$ planar arcs will be found among available distinct subsets when

$$
\left(\begin{array}{c}
N_{c} \\
m
\end{array}\right) \geq 1 / P_{\text {planar }}(m) \text {. }
$$

$m$ is maximized when the two sides are equal. Applying Sterling's approximation on both sides leads to $m_{\max } \propto$ $\sqrt{N_{c}}$. In other words, the typical number of pseudoknots for a random arc diagram approaches the number of contacts in the thermodynamic limit $\left(N_{c} \rightarrow \infty\right)$ as

$$
\left(N_{c}-\left\langle N_{p k}\right\rangle\right) / N_{c} \propto 1 / \sqrt{N_{c}} .
$$

Yet, true polymers and lattice walks do not come with random arc diagrams. There is a considerable correlation among the contacts due to the existence of an underlying chain and the effective repulsion resulting through self-avoidance, both of which favor contacts between monomers that are closer along the chain. This tendency is reflected in the loop length distribution [32],

$$
P(l) \propto l^{-c},
$$

where $c=d / 2$ (random-walk), $c=d \nu-\sigma_{4}=2.68,2.22$ (SAW in $2 \mathrm{~d}, 3 \mathrm{~d}$ ), and $\sigma_{4}$ the critical dimension associated with a 4-leg vertex as in the polymer network theory of Duplantier 27]. Correspondingly, one expects $N_{p k}$ for the real chains to be less than the above 'random graph' value. The combinatorial argument presented above picks random pairs of points along the chain with equal probability irrespective of the distance in between $(c=0)$. Unfortunately, it does not generalize easily to $c>0$. However, our numerical analysis on random graphs with arbitrary $c$ suggests

$$
\left\langle N_{c}-N_{p k}\right\rangle \propto N_{c}^{q(c)},
$$

with $q(c)$ increasing almost linearly from $q(0)=1 / 2$ and saturating at $q(\sim 2)=1$. The interesting statistical properties of the incompatibilty graph with the distribution in Eq.(3) will be reported elsewhere. A further reduction is expected in two dimensions due to additional constraints imposed by the impenetrability of encircled regions: The fact that each polymer contact divides the plane to two disconnected regions translates to having a bipartite incompatibility graph. As a consequence, $N_{p k} \leq N_{c} / 2$ (since the arc-diagram turns out to be planar when arcs are allowed on both half-planes instead of one). Unlike the general case, the vertex-cover problem on bipartite graphs is solvable in polynomial time 22.

One can obtain a lower-bound on $N_{p k}$ from Kesten's Pattern Theorem [23], i.e. by noting that a local pseudoknotted pattern (e.g. the shortest S-shaped walk on a square lattice) has a finite probability of occurence in an infinite chain. Therefore

$$
\left\langle N_{p k}\right\rangle>a N
$$

for a walk of $N$ steps and for some $a>0$. Extensivity of $\left\langle N_{p k}\right\rangle$ for a homopolymer supports the observation that their exclusion may have manifestations on the nature of the transition in the thermodynamic limit 13. and that penalizing pseudoknot formation can change the low-temperature phase from collapsed to a branched polymer (Section IV).

\section{PSEUDOKNOT NUMERICS}

In this part of our analysis we look at the ordinary homopolymer collapse, where the energy is not sensitive to the pseudoknot formation. We obtained statistics numerically for self-avoiding chains of typical size $N=300$, even though we checked for size independence of our results occasionally by going up to $N=800$. All our results were obtained by using an improved version of the PERM algorithm developed by Grassberger et.al. 24]. Although with PERM it is typical to simulate much longer homopolymers, our statistics were mainly limited by the fact that $N_{p k}$ for each configuration has to be calculated from scratch, unlike, e.g., the number of contacts which is updated incrementally at each step of the walk. We present results for square and cubic lattices in two and three dimensions, respectively.

It is possible to calculate $N_{p k}$ using an exact backtracking search algorithm which is straightforward to implement, but requires a runtime exponential in system size. Since we want to obtain statistics for reasonably long chains, it is not feasible to use such an exact method. Instead, we calculate $N_{p k}$ approximately by means of a greedy algorithm which at each step eliminates (one of) the maximally crossing $\operatorname{arc}(\mathrm{s})$. The choice is made randomly when they are more than one. Due to the stochastic nature of the algorithm and the fact that the optimal selection may involve eliminating a less than maximally crossing arc, this greedy algorithm provides an upper bound to $N_{p k}$. For details of various exact algorithms and the above greedy algorithm we refer the reader to [20]. 


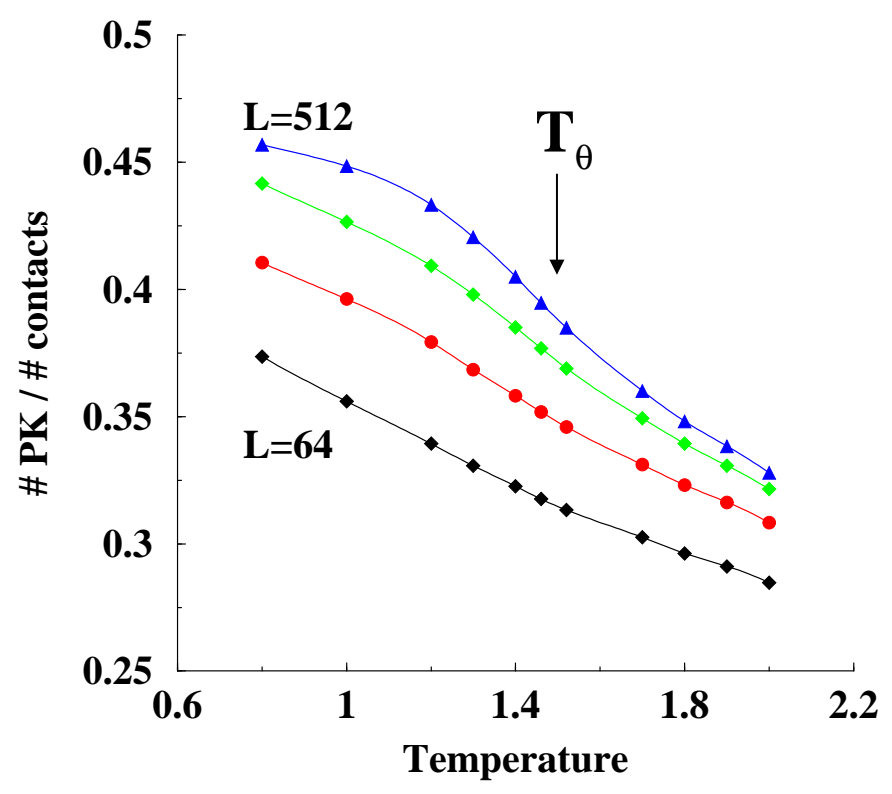

FIG. 3: Fraction of pseudocontacts in a SAW as a function of temperature for different walk lengths in two dimensions.

For comparison, we also implemented an exact calculation of $N_{p k}$ on the typical diagrams we encountered. We found that the average deviation from the exact value of the upper bound on $N_{p k}$ obtained by the greedy algorithm, although increasing with growing chain length, approaches a constant fraction around $1.5 \%$ of the exact $N_{p k}$. Therefore, we are confident that our conclusions concerning the scaling behavior are not effected by the approximate algorithm we adopted.

At all temperature regimes, we find that $\left\langle N_{p k}\right\rangle$ is a fraction of the total number of contacts with a temperature-dependent proportionality constant, $a(T)$. Although a Fermi-function-like limiting behavior is evident from Fig 3 the precise form of $a(T)$ in the thermodynamic limit requires a more elaborate analysis which we will not attempt here.

$a(T)$ reveals the leading behavior in $\left\langle N_{p k}\right\rangle$, however theory of critical phenomena has taught us that the nontrivial behavior of many systems is reflected in the nonanalytic contribution to the extensive quantities. Recently, Orlandini et.al. [25] considered the scaling of contacts formed between the two halves (referred from here on as $\mathrm{A}$ and $\mathrm{B}$ ) of a SAW at the $\theta$-point as a direct and precise way of measuring the crossover exponent, $\phi_{\theta}$. The number of such contacts scales as

$$
\left\langle N_{c}^{A B}\left(T_{\theta}\right)\right\rangle \propto N^{\phi_{\theta}},
$$

where $\phi_{\theta}=3 / 7$ in two dimensions, as can be shown analytically by using a recent extension of Saleur-Duplantier results for polymer criticality [26, 27]. The value of the crossover exponent is typically difficult to confirm by numerics, because, as a rule, it has to be extracted from subleading singular terms, when considring the set of all contacts along the chain 28]. Focusing on the contacts between the two halves strongly filters out the dominant analytical contribution of the local contacts along the chain, thus surfacing the otherwise concealed nonanalyticity. AB-contact statistics has been fruitful in a variety of polymer models [25, 29].

We use the same method here to pinpoint the singularity of $\left\langle N_{p k}\right\rangle$, which at the leading term scales identical to the contact energy (extensivity of $\left\langle N_{p k}\right\rangle$ due to Kesten's Pattern Theorem). More precisely, we start by identifying all A-B contacts, $N_{c}^{A B}$, as above, and then calculate the number of A-B pseudoknots, $N_{p k}^{A B}$, by eliminating the crossing arcs in the arc diagram corresponding to A-B contacts only. Since $\left\langle N_{p k}\right\rangle$ on the whole chain is a fraction of the contacts, $\left\langle N_{c}\right\rangle$, at all temperatures, one may expect that $\left\langle N_{p k}^{A B}\right\rangle$ should also scale identical to $\left\langle N_{c}^{A B}\right\rangle$ at all temparatures. In contrast, we find a qualitatively different scaling of the A-B pseudoknot number.

Let us consider each temperature regime separately:

Above the $\theta$-point $\left(T>T_{\theta}\right)$, no surprises are expected: $\left\langle N_{c}^{A B}\right\rangle$ already saturates to a constant in both two and three dimensions. Since $N_{p k}^{A B}<N_{c}^{A B}$, this leaves no alternative to $\left\langle N_{p k}^{A B}\right\rangle$ but to stay finite as well. This is confirmed by our numerics in Fig

At the $\theta$-point, the PERM algorithm is the most efficient. Therefore we expect our results to be most accurate in this region. Recall that $\left\langle N_{c}^{A B}\right\rangle \propto N^{3 / 7}$ for $T=T_{\theta}$, which can be obtained analytically and verified numerically to high accuracy. Our numerical results for $\left\langle N_{p k}^{A B}\right\rangle$ on the other hand indicate a logarithmic divergence of the form

$$
\left\langle N_{p k}^{A B}\left(T_{\theta}\right)\right\rangle \propto(\log N)^{v_{\theta}},
$$

where to our best estimate, $v_{\theta} \sim 3.9$ in two dimensions (Fig [5). In three dimensions, the logarithmic behavior survives (only at $T=T_{\theta}$ ), albeit with a different exponent $v_{\theta} \sim 4.3$.

In retrospect, one could argue a priori that $\left\langle N_{p k}^{A B}\right\rangle$ and $\left\langle N_{c}^{A B}\right\rangle$ should have qualitatively different scaling properties at the $\theta$-temperature:

We recall that the easiest way to obtain $\phi_{\theta}$ is to use the correspondence between a ring polymer at the $\theta$-point and the full hull of a percolating cluster at the percolation transition in two dimensions [25]. In this picture, the ABcontacts correspond to the the 'red' contacts between the two halves that lie between two diametrically opposite points of the hull (which are at the opposite infinities, see Fig 4a) 29].

The key observation is that, an AB-pseudoknot cannot be formed 'locally' between the two halves, because one of the two halves of the chain should wrap around the midpoint to make a pseudoknot-forming A-B contact, as shown in Fig $4 \mathrm{~b}$. This imposes a rather stringent 

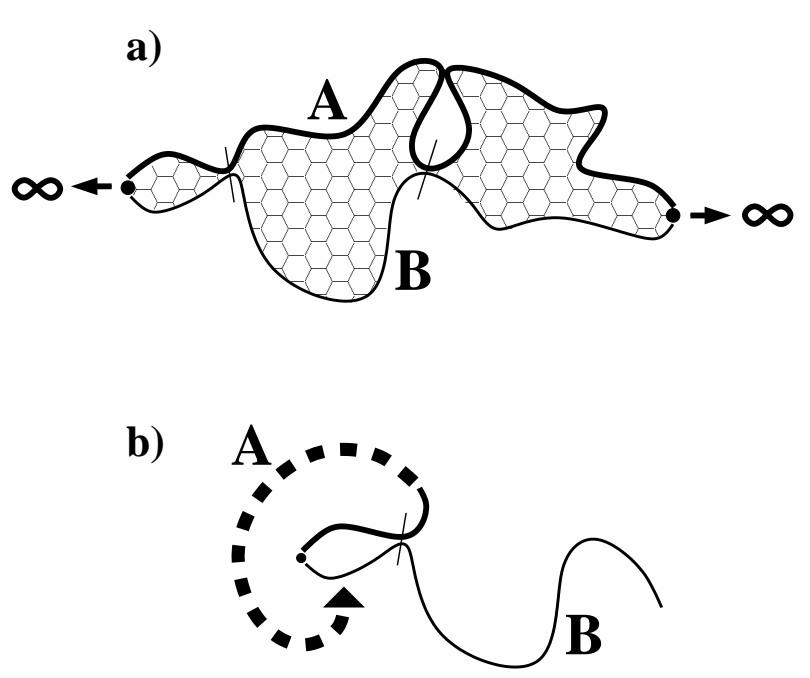

FIG. 4: The curves $\mathrm{A}$ (thick) and $\mathrm{B}$ (thin) represent the two halves of the full hull of a percolating cluster at the percolation transition. The number of $\mathrm{AB}$-contacts (indicated by the cutting line segments) scales as $N^{3 / 7}$ in the thermodynamic limit when the two diametrically opposite points on the hull bordering $\mathrm{A}$ and $\mathrm{B}$ are taken to infinity (a). An AB-pseudoknot can not be described in this setting once the thermodynamic limit is taken (b).

condition on the A-B pseudoknot formation. The numerical result of Eq. (6) nevertheless indicates a logarithmic divergence for $\left\langle N_{p k}^{A B}\right\rangle$. The scenario depicted in Fig $4 \mathrm{~b}$ suggests a likely connection to the statistics of the homopolymer winding angle, $\omega$, for which

$$
\left\langle\omega^{2}\right\rangle \propto \log N
$$

in the swollen phase and at the $\theta$-point in two dimensions [30]. Yet, this would have the interesting implication that the similar log-scaling observed in three dimensions has a different origin. These possibilities will be investigated in the future.

Below the $\theta$-point and in two dimensions, the logarithmic growth of $\left\langle N_{p k}^{A B}\right\rangle$ appears to persist. Although the numerics in this region is not as reliable, the lack of a power-law behavior similar to that for $\left\langle N_{p k}\right\rangle$ is not surprising due to the above geometric considerations. Note that the scaling of $\left\langle N_{c}^{A B}\right\rangle$ in this regime is still power-law 31]. In three dimensions and for $T<T_{\theta}^{3 d}$, preliminary calculations suggest $\left\langle N_{c}^{A B}\right\rangle \propto N$ and a deviation from the logarithmic behavior in $\left\langle N_{p k}^{A B}\right\rangle$. This is probably due to the fully A-B co-penetrated configurations of the compact polymer in $3 \mathrm{~d}$. Unlike in $2 \mathrm{~d}$, the A-B boundary fills the volume.

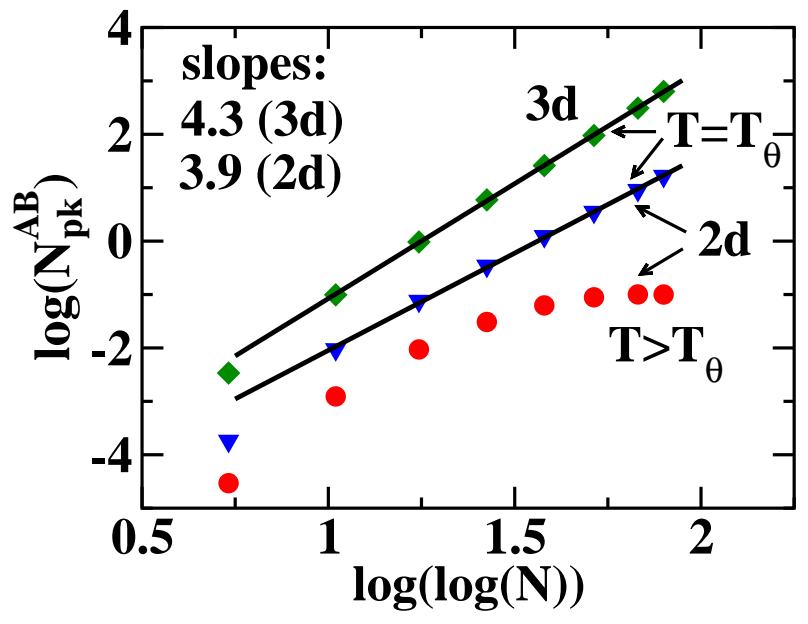

FIG. 5: Scaling of the A-B pseudoknot number for $T>T_{\theta}$ (2d), $T=T_{\theta}(2 \mathrm{~d})$ and $T=T_{\theta}(3 \mathrm{~d})$. Estimated asymptotic slopes are $v_{\theta} \sim 0,3.9$ and 4.3 , respectively. Maximum walk size was 800 steps in both dimensions.

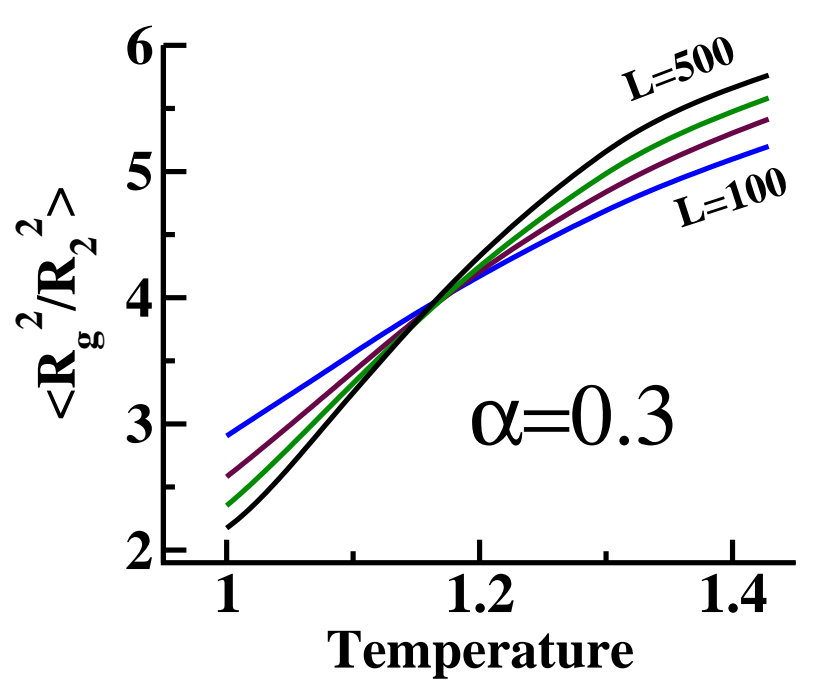

FIG. 6: Transition temperature for each value of $\alpha$ is located as the crossing point of $\left\langle R_{e}\right\rangle /\left\langle R_{g}\right\rangle$ curves as a function of temperature for different polymer lenghts. The curves is an interpolation between the data points for two dimensions and $\alpha=0.3$.

\section{PSEUDOKNOT-SENSITIVE HOMOPOLYMER COLLAPSE}

The pseudoknot formation is essential for the collapse of a polymer to a compact structure. This is most easily seen by comparing the radius of gyration, $R_{g}$, for a selfattracting homopolymer with that for which the pseudoknot forming contacts are excluded from the energy calculation. Consider the following generalized Hamilto- 
nian for a self-avoiding lattice walk:

$$
H=\sum_{i j} \epsilon_{c} \Delta(i, j)-\alpha \epsilon_{c} N_{p k}
$$

with $\Delta(i, j)=1$ for pairs of occupied nearest-neighbor lattice sites not consecutive along the walk and 0 else. $\alpha=0, \infty$ correspond respectively to the usual selfattracting homopolymer with and without pseudoknots. $\alpha=1$ is the case when the homopolymer energy is given by the number of contacts in the maximal planar 'sub'arc-diagram. In Eq.(8), we deliberately avoided writing down $N_{p k}$ as a sum over contacts, since only the number $N_{p k}$ and not the identity of the PK-forming contacts is well-defined.

We located the transition point for different values of $\alpha$ by plotting $\left\langle R_{e}\right\rangle /\left\langle R_{g}\right\rangle$, the ratio of the averaged end-toend distance and the radius of gyration $v s$. temperature. This universal ratio as a function of temperature should converge to a step function in the thermodynamic limit $(N \rightarrow \infty)$ with a universal intermediate value at the $\theta$ point 32, 33] The crossing point of the curves in Fig 6 for different chain sizes is an efficient way of locating the transition temperature and the critical universal ratio at the transition [13]. The resulting phase-diagram is given in Fig 7

\section{A. Phase Diagram}

Typical low-temperature configurations in the limit $\alpha \rightarrow \infty$ are double-stranded branched structures as shown in the inset of Fig[ In fact, one can show that the ground-state configurations in this limit are the nnn-avoiding lattice-trees. For a proof in two dimensions, it is sufficient to note that the number of energetically favorable contacts (size of the maximal planar sub-diagram) is maximized when the two ends of the walk meet at nearest-neighbor sites to form a fully deflated self-avoiding ring. Thus at zero temperature, the Hamiltonian in Eq. (8) with $\alpha=1$ is equivalent to the Leibler-Singh-Fisher (LSF) model 34] of planar vesicles with negative area fugacity. LSF model with negative pressure is established to have a BP low-temperature phase. The corresponding BP lives on the dual-lattice points inside the ring and the self-avoidence of the ring translates to next-nearest-neighbor-avoiding branches in the dual lattice. Consistently, we numerically obtain for the radius-of-gyration exponent, $\nu\left(T<T_{c}\right) \sim 0.62$, very close to the value of $\nu_{B P}=0.64$. SAW $\rightarrow \mathrm{BP}$ transition was studied earlier in several lattice polymer models 35, 36, 37]. We note that the Hamiltonian in Eq. (8) exhibits an SAW-BP transition also in higher dimensions, especially in three-dimensions which could be relevant to RNA-folding. The zero-temperature mapping to lattice trees presented above applies to three dimensions as well, although the dual lattice on which the corresponding branched polymers live is not simply the shifted simple cubic lattice.
The low-temperature scaling of $\left\langle R_{g}\right\rangle$ along the line $\alpha=$ 1 is still BP-like. Deep in the BP phase, $\epsilon^{p k} \equiv(\alpha-1) \epsilon_{c}$ acts as a contact interaction between the branches (negative $\alpha$ being the attractive regime). Considering earlier studies on BPs [38], we then expect a second transition line in each dimension between two low-temperature phases, $\mathrm{BP}$ and the collapsed polymer $(\mathrm{CP})$, as shown in dashed-line for two dimensions only in Fig 7 The simplest scenario is that the BP-CP boundary splits from the SAW-BP boundary at the $\theta$-point and asymptotically approaches the $\alpha=1$ line such that $\epsilon^{p k} / T=$ const. The critical interaction for the collapse of lattice-trees has been extensively studied in the literature [38, 39] for both dimensions. We merely speculate this section of the phase diagram, since obtaining good statistics at temperatures low enough to distinguish the two phases was not possible.

An interesting feature of the phase diagram in Fig[7 is the crossing of the phase boundaries corresponding to two and three dimensions around $\alpha=1$, reflecting the fact that the $T_{\theta}$ for a self-attracting homopolymer $(\alpha=0)$ increases with increasing dimension, whereas the $\mathrm{SAW} \rightarrow \mathrm{BP}$ transition temperature for $-\alpha \gg 1$ has the opposite trend! The transition temperature in each case is determined by the interplay between the entropy of the coil and the energy of the collapsed state. For $\alpha=0$, as we switch from the square to the cubic lattice, the increased gain in contact energy by collapse (due to the higher number of nearest-neighbor sites) overshadows the increased loss of entropy (due to -roughly- the change in the connectivity constant). As a result, $k_{B} T_{\theta} / \epsilon_{c}$ moves up from 1.5 to 3.5 . In the other limit $(-\alpha \gg 1)$, the contact energy due to the partial collapse to a BP is proportional to $\mathrm{N}$ and independent of dimension. Yet, the entropy loss due to collapse still increases with dimensionality. Then, the collapse to a BP should happen at a lower temperature with increasing dimension. The two opposing trends cancel each other around $\alpha=1$.

Also note that, positive but small values of $1-\alpha$ describe a transition upon reducing the temperature first to a branched-polymer-like state followed by a PK-mediated collapse. Such collapse (or melting) of RNAs with an intermediate pronounced with lowered $\mathrm{Mg}^{+2}$ concentration has been experimentally observed [17, 40, 41] and discussed in [15].

\section{CONCLUSIONS}

To summarize, we attempted in this paper to provide a mathematical definition for the number of pseudoknots, $N_{p k}$, in a polymer chain. With this definition, we show that counting the number of pseudoknots is equivalent to the well-known 'vertex-cover' problem which is NPcomplete. Nevertheless, it is possible to study numerically the statistical properties of pseudoknots by employing an efficient approximate scheme. We show that the average total number of pseudoknots is extensive at all 


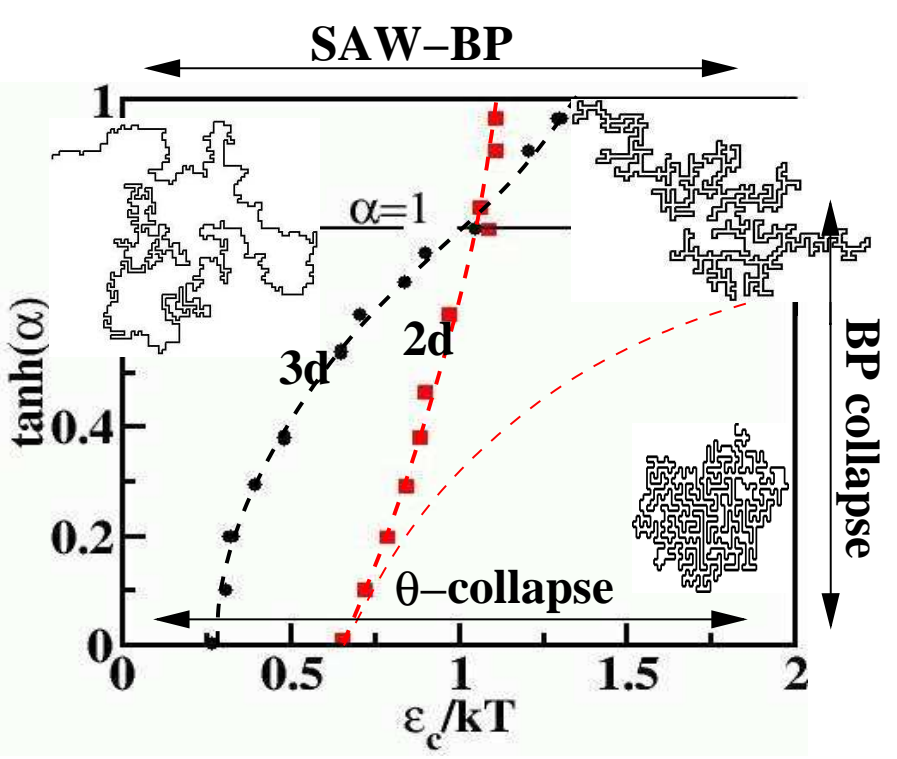

FIG. 7: The phase-diagram for the Hamiltonian in Eq 8 The lower horizontal axis corresponds to the usual homopolymer with self-attraction. SAW-BP transition line were obtained from the numerical data by the universal-ratio crossing method described in the text. The terminal point on the lower axis of the transition curves correspond to the $\theta$-point for that dimension. Dashed curve is the expected BP-CP boundary with a limiting $(\mathrm{T} \rightarrow 0)$ value of $(1-\alpha) / T=0.69$. temperatures, however the number of pseudoknots forming between the two-halves of the chain scales logarithmically with chain size at the $\theta$-point of a homopolymer in both two and three dimensions, and also for $T<T_{\theta}$ in two dimensions. This logarithmic character is likely be related to the winding-angle statistics in two dimensions.

We also studied the role of pseudoknots in the homopolymer collapse by considering a Hamiltonian which favors polymer self-contacts but penalizes pseudoknots. We showed that in the absence of an energetic preference for pseudoknot formation, the low-temperature phase is a branched-polymer. When the ratio of two competing energies satisfies $0<\alpha<1$, Hamiltonian (8) allows a transition scenario from a SAW to a collapsed phase with an intermediate branched polymer regime, where the BP$\mathrm{CP}$ transition is mediated by pseudoknot formation. The critical properties of the phase diagram and relevance to RNA folding need further investigation.

\section{ACKNOWLEDGEMENTS}

A.K. thanks D. Yuret and C.E. Soteros for pointing out, respectively, the NP-complete character of the problem and the existence of a polynomial-time solution on bipartite graphs. We acknowledge support from INFMPA02 and MIUR-COFIN01.
[1] E.B. ten Dam, C.W.A. Pleij, and D. Draper, Biochemistry 31, 11665 (1992); C.W.A. Pleij, Curr. Opin. Struc. Biol. 4, 337 (1994).

[2] A. Xayaphoummine, T. Bucher, F. Thalmann, and H. Isambert, cond-mat 0309117

[3] D.S. McPheeters, G.D. Stormo, and L. GOld, J. Mol. Biol. 201, 517 (1990).

[4] Z. Du, J.A. Holland, M.R. Hansen, D.P. Giedroc, and D.W. Hoffman, J. Mol. Biol. 271, 463 (1997).

[5] N.M. Wills, R.F. Gesteland, and J.F. Atkins, Proc. Natl. Acad. Sci. 88, 6991 (1991).

[6] P.J. Farabaugh, Cell 74, 591 (1993).

[7] R. Nussinov and A.B. Jacobson, Proc. Nat. Acad. Sci. 77, 6309 (1980).

[8] M. Zuker and P. Stiegler, Mucl. Acids Res. 9, 133 (1981).

[9] R. Bundschuh and T. Hwa, Phys. Rev. Lett. 83, 1479 (1999).

[10] E. Rivas, S. Eddy, J. Mol. Biol. 285, 2053 (1999).

[11] Y. Uemura, A. Hasegawa, S. Kobayashi, and T. Yokomori, Theo. Comp. Sci. 210, 277 (1999).

[12] M. Pilsbury, J.A. Taylor, H. Orland, and A. Zee, cond-mat/0310505

[13] M. Baiesi, E. Orlandini, and A.L. Stella, Phys. Rev. Lett 87, 070602 (2003).

[14] A. Lucas and K.A. Dill, J. Chem. Phys. 119, 2414 (2003).

[15] P. Leoni and C. Vanderzande, Phys. Rev. E. 68, 051904 (2003).

[16] There is experimental evidence that these regimes can be reached by modifying the $\mathrm{Mg}^{+2}$ concentration in the solution. E.g., see V.P. Misra and D.E. Draper, Biopolymers 48, 113 (1999).

[17] I. Tinoco Jr. and C. Bustamante, J. Mol. Biol. 293, 271 (1999).

[18] M. Vendruscolo, B. Subramanian, I. Kanter, E. Domany, and J. Lebowitz, Phys. Rev. E 59, 977 (1999).

[19] M.R. Garey and D.S. Johnson, Computers and Intractability: A Guide to the Theory of NP-Completeness, W.H. Freeman \& Co. (1979).

[20] M. Weigt and A.K. Hartmann, Phys. Rev. E 63, 056127 (2001).

[21] R.B. Lyngso and C.N.S. Pedersen, J. Comp. Biol. 7, 409 (2000).

[22] G. Chartrand and O. Oellermann, "Applied and Algorithmic Graph Theory", McGraw-Hill (1992).

[23] N. Madras, G. Slade, The Self-Avoiding Walk, Birkhäuser Press (1996).

[24] H.-P. Hsu, V. Mehra, W. Nadler, and P. Grassberger, J. Chem. Phys 118, 444 (2003).

[25] E. Orlandini, F. Seno, A.L. Stella, Phys. Rev. Lett. 84, 294 (2000).

[26] M. Aizenman, B. Duplantier, and A. Aharony, Phys. Rev. Lett.83, 1359 (1999).

[27] B. Duplantier J. Stat. Phys. 54, 581 (1988).

[28] The connection between the crossover exponent and the sub-dominant factor in the free energy was recently emphasized to explain certain numerical results. See A.L. Owczarek, T. Prellberg, Phys. Rev. E 67, 032801 (2003).

[29] M. Baiesi, E. Carlon, E. Orlandini, A.L. Stella, Phys. 
Rev. E. 63, 041801 (2001).

[30] B. Drossel and M. Kardar, Phys. Rev. E 53, 5861 (1996).

[31] A naive argument based on a mapping of the lowtemperature AB-contacts to high-temperature SAW suggests an exponent $\phi=2 / 3$ (unpublished).

[32] C. Vanderzande, Lattice Models of Polymers, Cambridge University Press (1996).

[33] V. Privman, P.C. Hohenberg, and A. Aharony in Phase Transitions and Critical Phenomena, edited by C. Domb and J.L. Lebowitz, Academic Press, New York (1991), vol. 14 p. 1.

[34] S. Leibler, R.R.P. Singh, and M.E. Fisher, Phys. Rev. Lett. 59, 1989 (1987).
[35] E. Orlandini, F. Seno, A.L. Stella, and M.C. Tesi, Phys. Rev. Lett. 68, 488 (1992).

[36] R. Dekeyser, E. Orlandini, A.L. Stella, and M.C. Tesi, Phys. Rev. E 52, 5214 (1995).

[37] A.L. Stella, Phys. Rev. E 50, 3259 (1994).

[38] F. Seno and C. Vanderzande, J. Phys. A 27, 5813 (1994).

[39] N. Madras, E.J. Janse van Rensburg, J. Stat. Phys. 86, 1 (1997).

[40] D.K. Treiber, M.S. Rook, P.P. Zarrinkar, and J.R. Williamson, Science 279, 1943 (1998).

[41] J. Pan and S.A. Woodson, J. Mol. Biol. 280, 597 (1998). 